CECS-PHY-07/14

\title{
Black holes, parallelizable horizons and half-BPS states for the Einstein-Gauss-Bonnet theory in five dimensions
}

\author{
Fabrizio Canfora ${ }^{1,2}$, Alex Giacomini ${ }^{1}$ and Ricardo Troncoso ${ }^{1}$ \\ ${ }^{1}$ Centro de Estudios Cientificos (CECS), Casilla 1469 Valdivia, Chile. \\ ${ }^{2}$ Istituto Nazionale di Fisica Nucleare, Sezione di Napoli, GC Salerno. \\ e-mail: canfora@cecs.cl, giacomini@cecs.cl, ratron@cecs.cl
}

\begin{abstract}
Exact vacuum solutions with a nontrivial torsion for the Einstein-Gauss-Bonnet theory in five dimensions are constructed. We consider a class of static metrics whose spacelike section is a warped product of the real line with a nontrivial base manifold endowed with a fully antisymmetric torsion. It is shown requiring solutions of this sort to exist, fixes the Gauss-Bonnet coupling such that the Lagrangian can be written as a Chern-Simons form. The metric describes black holes with an arbitrary, but fixed, base manifold. It is shown that requiring its ground state to possess unbroken supersymmetries, fixes the base manifold to be locally a parallelized threesphere. The ground state turns out to be half-BPS, which could not be achieved in the absence of torsion in vacuum. The Killing spinors are explicitly found.
\end{abstract}

\section{Introduction}

Nowadays it is widely accepted by the high energy physics community that the search for a unified theory seems to require additional spacetime dimensions. In five dimensions, if one follows the basic principles of General Relativity, the most general theory of gravity leading to second order field equations for the metric is described by the so called EinsteinGauss-Bonnet action [1]

$$
I=\kappa \int d^{5} x \sqrt{g}\left(R-2 \Lambda+\tilde{\alpha}\left(R^{2}-4 R_{\mu \nu} R^{\mu \nu}+R_{\alpha \beta \gamma \delta} R^{\alpha \beta \gamma \delta}\right)\right),
$$


where $\kappa$ is related to the Newton constant, $\Lambda$ to the cosmological term, and $\tilde{\alpha}$ is the Gauss-Bonnet coupling. For later convenience, it is useful to express the action (11) in terms of differential forms as 1

$$
I=\int \epsilon_{a b c d e}\left(\frac{c_{0}}{5} e^{a} e^{b} e^{c} e^{d} e^{e}+\frac{c_{1}}{3} R^{a b} e^{c} e^{d} e^{e}+c_{2} R^{a b} R^{c d} e^{e}\right),
$$

where $e^{a}=e_{\mu}^{a} d x^{\mu}$ is the vielbein, and $R^{a b}=d \omega^{a b}+\omega_{c}^{a} \omega^{c b}$ is the curvature 2-form for the spin connection $\omega^{a b}=\omega_{\mu}^{a b} d x^{\mu}$.

In the first order formalism, the action (2) is extremized varying both with respect to the vielbein and with respect to the spin connection independently, so that the field equations read

$$
\mathcal{E}_{e}:=\left(c_{o} e^{a} e^{b} e^{c} e^{d}+c_{1} R^{a b} e^{c} e^{d}+c_{2} R^{a b} R^{c d}\right) \epsilon_{a b c d e}=0
$$

and

$$
\mathcal{E}_{a b}:=T^{c}\left(c_{1} e^{d} e^{e}+2 c_{2} R^{d e}\right) \epsilon_{a b c d e}=0,
$$

respectively. The torsion is defined as the covariant derivative of the vielbein, i.e., $T^{a}=$ $D e^{a}$.

In the vanishing torsion sector, the field equations (4) are trivially fulfilled, and Eq. (3) reduces to the standard one in the second order formalism. Nevertheless, one peculiar feature of the Einstein-Gauss-Bonnet theory, which distinguish it from standard General Relativity, is that the field equations (44) do not necessarily imply the vanishing of torsion [2], so that in the first order formalism, besides the graviton, there are additional propagating degrees of freedom related to the torsion. Generically, the field equations (4) impose very strong constraints on the torsion; however, this situation can be softened for certain values of the Gauss-Bonnet coupling. For instance, requiring the theory to posses the maximum number of degrees of freedom, fixes the Gauss-Bonnet coupling as [2]

$$
c_{2}=\frac{c_{1}^{2}}{4 c_{0}}
$$

so that the theory possesses a unique maximally symmetric vacuum [3], and the Lagrangian can be written as a Chern-Simons form [4]. For the choice (5) some exact solutions with torsion in vacuum have been found [5]. Further choices of the GaussBonnet coupling also allow the existence of torsion in vacuum, for which the number of degrees of freedom is not the maximum. Explicit solutions of this sort have been recently found in [6], which have the structure of a cross product of a two-dimensional Riemannian manifold of Lorentzian signature and constant curvature, with an Euclidean tree-dimensional manifold of constant curvature and nonvanishing fully antisymmetric

\footnotetext{
${ }^{1}$ The relationship between the constants appearing in Eqs (11) and (2) is given by $\tilde{\alpha}=\frac{c_{2}}{2 c_{1}}, \Lambda=-6 \frac{c_{0}}{c_{1}}$, $\kappa=2 c_{1}$. Moreover, for notational simplicity, the wedge product between forms is understood.
} 
torsion. Thus, it is natural to wonder whether there exists a black hole solution of the five-dimensional Einstein-Gauss-Bonnet theory whose horizon geometry is described by a three-dimensional manifold endowed with a fully antisymmetric torsion.

In this paper, exact vacuum solutions with a nontrivial torsion for the Einstein-GaussBonnet theory in five dimensions are constructed. We consider a class of static metrics of the form

$$
d s^{2}=-f^{2}(r) d t^{2}+\frac{d r^{2}}{f^{2}(r)}+r^{2} d \Sigma_{3}^{2}
$$

where $d \Sigma_{3}^{2}$ stands for the metric of a three-dimensional base manifold $\Sigma_{3}$, which is endowed with a fully antisymmetric torsion. In the next Section it is shown that requiring solutions of this sort to exist, fixes the Gauss-Bonnet coupling as in Eq. (5) , and the metric describes black holes with an arbitrary, but fixed, base manifold $\Sigma_{3}$. In Section 3 we show that requiring the black hole to have a ground state possessing unbroken supersymmetries, fixes the base manifold to be locally a parallelized three-sphere. It is worth pointing out that unlike the Riemannian case, which is devoid of torsion, this ground state turns out to be half-BPS, and their Killing spinors are explicitly found. Finally, Section 4 is devoted to the discussion and comments.

\section{Black holes with nontrivial torsion}

Let us now search for an exact solution of the field equations (3) and (4), whose metric is of the form (6) . The vielbein can then be chosen as

$$
e^{0}=f(r) d t ; \quad e^{1}=f^{-1}(r) d r ; \quad e^{m}=r \tilde{e}^{m},
$$

where $\tilde{e}^{m}$ stands for the vielbein of the base manifold, so that latin indices $m, n, p, \ldots$ run along $\Sigma_{3}$.

The torsion is assumed to be fully antisymmetric, static, and such that its only nonvanishing components have support only along the base manifold. A simple ansatz in this case is given by

$$
T^{m}=K(r) \epsilon^{m n p} e_{m} e_{p}
$$

It is useful to define the contorsion one-form $K^{a b}$, which fulfils $T^{a}=K^{a b} e_{b}$, so that the spin connection splits into its Riemannian and non Riemannian parts according to

$$
\omega^{a b}=\stackrel{\circ}{\omega}^{a b}+K^{a b}
$$

where $\stackrel{\circ}{\omega}^{a b}$ is the Levi-Civita (torsion-free) spin connection, and the curvature two-form can be written as

$$
R^{a b}=\stackrel{\circ}{R}^{a b}+\stackrel{\circ}{D} K^{a b}+K_{c}^{a} K^{c b}
$$

where $\stackrel{\circ}{R}^{a b}=d \stackrel{\circ}{\omega}^{a b}+\stackrel{\circ}{\omega}_{c}^{a} \stackrel{o}{\omega}^{c b}$ is the Riemannian curvature, and $\stackrel{\circ}{D}$ is the covariant derivative for the Levi-Civita spin connection. 
Hence, by virtue of Eq. (8), the only non vanishing components of the contorsion are given by

$$
K^{m n}=-K(r) \epsilon^{m n p} e_{p},
$$

so that the spin connection for the ansatz (7), and (8) reads

$$
\omega^{01}=f^{\prime} e^{0} ; \quad \omega^{n 1}=f(r) \tilde{e}^{n} ; \quad \omega^{m n}=\tilde{\omega}^{m n}+K^{m n},
$$

where $\tilde{\omega}^{m n}$ corresponds to the Levi-Civita connection of the base manifold.

The Riemannian curvature reads

$$
\begin{gathered}
\stackrel{\circ}{R}^{01}=-\frac{\left(f^{2}\right)^{\prime \prime}}{2} e^{0} e^{1} \quad ; \quad \stackrel{\circ}{R^{0 n}}=-\frac{\left(f^{2}\right)^{\prime}}{2 r} e^{0} e^{n} \\
\stackrel{\circ}{R}^{1 n}=-\frac{\left(f^{2}\right)^{\prime}}{2 r} e^{1} e^{n} \quad ; \quad \stackrel{\circ}{R}^{m n}=\tilde{R}^{m n}-\frac{f^{2}}{r^{2}} e^{m} e^{n}
\end{gathered}
$$

where $\tilde{R}^{m n}$ stands for the curvature of the base manifold $\Sigma_{3}$, so that by virtue of (10), the curvature two-form is given by

$$
\begin{gathered}
R^{01}=\stackrel{\circ}{R}^{01} ; R^{0 m}=\stackrel{\circ}{R}^{0 m} ; \quad R^{1 n}=\stackrel{\circ}{R}^{1 n}-\frac{f}{r} T^{n}, \\
R^{m n}=\stackrel{\circ}{R}^{m n}-\frac{d(r K)}{r} \epsilon^{m n p} e_{p}-K^{2} e^{m} e^{n} .
\end{gathered}
$$

Let us begin solving the field equations (4) assuming a nonvanishing torsion.

Due to the form of our ansatz (66) and (18), the components $\mathcal{E}_{1 m}=0$, and $\mathcal{E}_{0 m}=0$ of the field equations (4) are identically fulfilled. The components of (4) along the base manifold, i.e., $\mathcal{E}_{m n}=0$ are solved provided $\left(f^{2}\right)^{\prime \prime}=c_{1} / c_{2}$, which means that the function $f^{2}(r)$ is of the form

$$
f^{2}=\frac{c_{1}}{2 c_{2}} r^{2}+\alpha r-\mu
$$

The remaining equation $\mathcal{E}_{01}=0$, is solved provided $d(r K)=0$, which fixes the form of the function $K(r)$ in Eq. (8) as

$$
K=-\frac{\delta}{r}
$$

where $\delta$ is an integration constant.

Let us now focus on the field equations (3).

The radial component of (3), $\mathcal{E}_{1}=0$, reduces to

$$
6 A(r)+\tilde{R} B(r)=0,
$$


where $\tilde{R}$ is the Ricci scalar of the base manifold, and

$$
\begin{aligned}
& A=4 c_{0}-\frac{c_{1}^{2}}{c_{2}}+2 c_{2} \alpha\left(\frac{\delta^{2}-\mu}{r^{3}}+\frac{\alpha}{r^{2}}\right), \\
& B=-2 \frac{c_{2} \alpha}{r} .
\end{aligned}
$$

Since $\tilde{R}$ depends only on the coordinates of the base manifold, Eq. (17) implies that

$$
A+\gamma B=0
$$

where $\gamma$ is a constant.

Hence, Eq. (18) implies that the constant $\alpha$ vanishes, and that the Gauss-Bonnet coupling must necessarily be fixed as in Eq. (5). Furthermore, note that Eq. (17) does not impose any restriction on the base manifold $\Sigma_{3}$. Using this, it is easy to verify that the constraint $\mathcal{E}_{0}=0$, as well as the remaining field equations $\mathcal{E}_{m}=0$ are fulfilled.

In sum, the solution describes an asymptotically AdS spacetime whose metric reads

$$
d s^{2}=-\left(\frac{r^{2}}{l^{2}}-\mu\right) d t^{2}+\frac{d r^{2}}{\frac{r^{2}}{l^{2}}-\mu}+r^{2} d \Sigma_{3}^{2},
$$

where $l=\sqrt{\frac{2 c_{2}}{c_{1}}}$ is the AdS radius 2 , possessing a non vanishing torsion whose only nonvanishing components are given by

$$
T^{m}=-\frac{\delta}{r} \epsilon^{m n p} e_{m} e_{p}
$$

For this solution, the torsion and the Riemannian curvature are singular at the origin, but nevertheless these singularities are surrounded by an event horizon located at $r^{2}=\mu l^{2}$, so that this geometry describes a black hole whose horizon geometry is endowed with a nontrivial torsion. $\Sigma_{3}$

It is worth to remark that the field equations are solved for any fixed base manifold

In the absence of torsion, this solution has been recently shown to exist for the EinsteinGauss-Bonnet theory only if the coefficients are fine tuned as in Eq. (5) [7]. For base manifolds of constant curvature the torsionless solution reproduces the ones found in [8], and [9], which in the case of spherical symmetry reduces to the one in [10], [11] $]^{3}$.

\footnotetext{
${ }^{2}$ The asymptotically dS solution is obtained just making $l \rightarrow i l$. In this case the solution generically possesses a cosmological horizon and a timelike naked (Riemannian) curvature singularity at the origin. The torsion also diverges at the origin, even in the case $\mu=-1$, for which the metric reduces to de Sitter spacetime. We no longer discuss this case here.

${ }^{3}$ For a generic value of the Gauss-Bonnet coupling $c_{2}$, the solution with a base manifold of constant curvature in the absence of torsion has been found in [12].
} 
It is worth pointing out the similarity of the black hole solution found here with the BTZ black hole [13], [14]. In three dimensions the static solution describes a black hole provided $\mu>0$, and $\mu=0$ is the "black hole vacuum". For the range $-1<\mu<0$, the solution has a naked conical singularity, and for $\mu=-1$ the metric is that of $A d S_{3}$. For the static case, the only solutions with unbroken supersymmetries within this family are $A d S_{3}$, being maximally supersymmetric, and the zero mass black hole which has two Killing spinors [15], so that it corresponds to the ground state of (1,1)-AdS supergravity [16] with periodic (Ramond) boundary conditions on the spinor fields.

In the case of the five-dimensional black hole without torsion, the situation is almost analogous to the three-dimensional case, since the solution for $\mu=-1$ corresponds to $A d S_{5}$ spacetime, while for the range $-1<\mu<0$, it describes timelike naked singularities. The black hole is also obtained for $\mu>0$, and the solution with $\mu=0$ corresponds to the black hole vacuum. However, it has been shown that in the absence of torsion and matter fields, the only solution possessing Killing spinors within this family is the maximally supersymmetric $A d S_{5}$ spacetime, so that the zero mass black hole breaks all the supersymmetries [17].

Hence, it is natural to wonder whether the presence of torsion helps to improve the situation in five dimensions, in the sense that if the black hole vacuum with torsion had Killing spinors, its stability would be guaranteed preventing the black hole from decaying into naked singularities.

In the next section it is shown that for the black hole solution given by Eqs. (19) and (20), which possesses an arbitrary but fixed base manifold, requiring its groundstate to possess unbroken supersymmetries, removes the arbitrariness in the base manifold since $\Sigma_{3}$ becomes fixed to be locally a parallelized (combed) three-sphere. It is worth to remark that unlike the standard torsion-free case, this ground state turns out to be half-BPS.

\section{$3 \quad$ Half-BPS ground state and locally parallelizable $S^{3}$ horizon}

The locally supersymmetric extension of the Einstein-Gauss-Bonnet theory in five dimensions with the choice of couplings as in (5) is known [18. The field equations as well as the local supersymmetry transformations can be explicitly found in [19] (see also [20]). It is simple to prove that our solution, given by Eqs. (19) and (20), solves the field equations of supergravity in the absence of matter fields.

The Killing spinor equation is obtained requiring a purely bosonic configuration to possess unbroken global supersymmetries. In the absence of matter fields, i.e., for the purely gravitational sector, the Killing spinor equation is given by

$$
\nabla \epsilon:=\left(d+\frac{1}{4} \omega^{a b} \Gamma_{a b}+\frac{1}{2 l} e^{a} \Gamma_{a}\right) \epsilon=0
$$


where $\Gamma_{a b}$ is expressed in terms of Dirac matrices as $\Gamma_{a b}=(1 / 2)\left[\Gamma_{a}, \Gamma_{b}\right]$. The consistency condition of Eq. (21) reads

$$
\nabla \nabla \epsilon=\left(\frac{1}{4}\left(R^{a b}+\frac{1}{l^{2}} e^{a} e^{b}\right) \Gamma_{a b}+\frac{1}{2 l} T^{a} \Gamma_{a}\right) \epsilon=0 .
$$

For the black hole solution given by Eqs. (19) and (20), where the local frame has been chosen as in Eq. (7), the consistency condition turns out to be

$$
\left[\frac{1}{2}\left(\tilde{R}^{m n}+\left(\mu-\delta^{2}\right) \tilde{e}^{m} \tilde{e}^{n}\right) \Gamma_{m n}+r \delta \epsilon^{m n p} \tilde{e}_{n} \tilde{e}_{p}\left(\frac{1}{l}-\sqrt{\frac{1}{l^{2}}-\frac{\mu}{r^{2}}} \Gamma_{1}\right) \Gamma_{m}\right] \epsilon=0 .
$$

Note that there is no way to collect terms having the same functional dependence on $r$ unless $\mu=0$. In this case the consistency condition reduces to

$$
\left[\frac{1}{2}\left(\tilde{R}^{m n}-\delta^{2} \tilde{e}^{m} \tilde{e}^{n}\right) \Gamma_{m n}+\frac{r}{l} \delta \epsilon^{m n p} \tilde{e}_{n} \tilde{e}_{p} \Gamma_{m}\left(1+\Gamma_{1}\right)\right] \epsilon=0
$$

which admits nontrivial solutions provided the Killing spinor is an eigenstate of $\Gamma_{1}$, i.e.,

$$
\Gamma_{1} \epsilon=-\epsilon
$$

Hence, Eq. (24) reduces to a condition on the Riemaniann curvature of the base manifold given by

$$
\left(\tilde{R}^{m n}-\delta^{2} \tilde{e}^{m} \tilde{e}^{n}\right) \Gamma_{m n} \epsilon=0 .
$$

Since the base manifold $\Sigma_{3}$ is of Euclidean signature, this last equation implies that $\Sigma_{3}$ must be locally a space of positive constant curvature, whose radius is fixed in terms of the strength of the torsion as

$$
\tilde{R}^{m n}=\delta^{2} \tilde{e}^{m} \tilde{e}^{n} .
$$

Therefore, as the base manifold describes the geometry of the horizon, orientability and smoothness has to be required. Condition (27) then fixes the Riemannian geometry of $\Sigma_{3}$ to be that of any smooth and orientable quotient of the three-sphere $S^{3}$, which are fully classified (see e. g. [21]). A simple example corresponds to the real projective space $R P^{3}$. Furthermore, as it can be seen from Eq. (14), in this case the curvature two-form of the base manifold vanishes, and hence, the base manifold is fixed to be locally a parallelized three-sphere. Thus, the vielbeins of the base manifold can be chosen locally as $\tilde{e}^{m}=\sigma^{m}$, where $\sigma^{m}$ stand for the left-invariant forms of $S U(2)$, satisfying $d \sigma^{m}=-\epsilon^{m n p} \sigma_{n} \sigma_{p}$, so that the Levi-Civita spin connection of $\Sigma_{3} \operatorname{reads} \tilde{\omega}^{m n}=-\epsilon^{m n p} \tilde{e}_{p}$.

Note that for the generic black hole solution given by (19) and (20) the constant $\delta$ is arbitrary. However, the condition (27) implies that $\delta$ cannot longer be "a truly" 
integration constant, since it can be brought into the form $\delta=1$ under suitable rescalings of time and radial coordinates. 4 .

Let us solve the Killing spinor equation (21). The consistency condition fixes $\mu=0$, so that $f=r / l$, and as the base manifold becomes parallelized, its intrinsic spin connection vanishes (see Eq. (12) ). Furthermore, using the chirality condition (25), and reading the vielbein and the remaining components of the spin connection from Eqs. (7), (9) , (20) with $\delta=1$, and (12), respectively, the Killing spinor equation reduces to

$$
\left(d-\frac{1}{2 r} d r\right) \epsilon=0 .
$$

Therefore, the Killing spinor does not depend neither on time nor on the coordinates of the base manifold (since $\partial_{t} \epsilon=\partial_{m} \epsilon=0$ ). The Killing spinors are given by the solution radial equation

$$
\left(\partial_{r}-\frac{1}{2 r}\right) \epsilon=0
$$

which can be integrated as

$$
\epsilon=\sqrt{\frac{r}{l}} \eta_{0}
$$

where $\eta_{0}$ is a constant spinor satisfying the chirality condition

$$
\Gamma_{1} \eta_{0}=-\eta_{0}
$$

Therefore, unlike for the torsion-free case, one concludes that the black holes described by Eqs. (19) and (20), which generically have an arbitrary but fixed base manifold, possess a half-BPS groundstate only for base manifolds which are locally a parallelized (combed) three-sphere with a torsion given by

$$
T^{m}=-\frac{1}{r} \epsilon^{m n p} e_{m} e_{p}
$$

The supersymmetry of the groundstate, which would guarantee its stability, then prevents a black hole with a torsion given by (32) from decaying into naked singularities. The Killing spinors are explicitly given by Eq. (30) with the chirality condition (31) 5 .

\footnotetext{
${ }^{4}$ The case $\delta=-1$ is connected to the case $\delta=1$ trough a full reflection of the local frames of the base manifold. It is then convenient to choose $\delta=1$, so that the base manifold can be manifestly paralellized making its intrinsic spin connection to vanish.

${ }^{5}$ In view of the highly non-trivial effect of the torsion, it would be interesting to compute its coupling with the spin current as done in 26]
} 


\section{Discussion and comments}

In this paper we have found an exact black hole solution in vacuum with nontrivial torsion for the Einstein-Gauss-Bonnet theory in five dimensions. The metric is given by Eq. (19), and this spacetime is endowed with torsion as in Eq. (20). It was shown that requiring solutions of this sort to exist, fixes the Gauss-Bonnet coupling as in Eq. (5), such that the Lagrangian can be written as a Chern-Simons form. The metric describes black holes with an arbitrary, but fixed, base manifold, and it is such that in the torsion-free the solution reduces to the one found in [7].

It is simple to prove that the black hole with torsion solves the supergravity field equations in the absence of matter fields 6 . Thus, it was shown that requiring the black hole to have a groundstate with unbroken supersymmetries, fixes the integration constant of the torsion as in Eq. (32), so that the base manifold is constrained to be locally a parallelized three-sphere. It is worth to remark that unlike the standard torsion-free case, this ground state turns out to be half-BPS, which would guarantee its stability, preventing a black hole with a torsion given by (32) from decaying into naked singularities. The Killing spinors are given explicitly by Eq. (30) with the chirality condition (31).

It is worth to remark that the possibility of having a non trivial torsion in vacuum may have a very important role in stabilizing other classes of solutions which otherwise would be unstable.

Note that when the Gauss-Bonnet coupling is chosen as (15) the theory not only acquires propagating degrees of freedom for the torsion, but it is also invariant under a local $A d S$ boosts [2], so that the curvature and the torsion transform into each other in a non trivial way. Thus, a torsionless solution can be transformed into another one with torsion given by

$$
\delta T^{a}=\left(R^{a b}+\frac{1}{l^{2}} e^{a} e^{b}\right) \lambda_{b}
$$

where $\lambda_{b}$ is the $A d S$ boost parameter, which is not diffeomorphically equivalent to the former.

In this sense the black hole solution found here possesses an intrinsic torsion, since it cannot be gauged away with an $A d S$ boost.

It is worth pointing out that the ansatz for the torsion (8) has been inspired by an analogy between spacetimes with nontrivial torsion and BPS states in Yang-Mills theory [25], which suggests the existence of further possibilities to find exact solutions with intrinsic torsion. It would also be interesting to see whether this kind of torsion contributes to the spin current such that it could affect the chiral anomaly as in Ref. [26].

It is also natural to wonder whether there are black holes with a non trivial torsion in vacuum for dimensions other than five. It has been recently shown that three-dimensional

\footnotetext{
${ }^{6}$ Exact solutions of the supergravity field equations with a Gauss-Bonnet coupling of the form (5), and with nonvanishing matter fields can be found in Refs. 22., 23, 20], 24].
} 
supergravity can be extended to the case in which the gravitational sector admits torsion in vacuum [27], so that the BTZ black hole with torsion [28] solves the field equations in vacuum. Thus, the Killing spinors of the extremal cases of the BTZ black hole with torsion can be obtained from the ones of the BTZ black hole without torsion [15], by means of a simple map introduced in [27].

It would be interesting to explore the possibility of having unbroken supersymmetries in dimensions higher than five with non trivial torsion in vacuum, which is allowed for the class of supergravity theories constructed in Refs. [29], and [19].

Acknowledgments.- We thank J. Oliva for useful remarks. Special acknowledge to Steve Willison, who participated in an early stage of this work, for helpful comments. F.C. thanks PRIN SINTESI 2007 for financial support. This work was partially funded by FONDECYT grants 1051056, 1061291, 1071125, 3060016, 3070055, 3070057. The generous support to Centro de Estudios Científicos (CECS) by Empresas CMPC is also acknowledged. CECS is funded in part by grants from the Millennium Science Initiative, Fundación Andes and the Tinker Foundation.

\section{References}

[1] D. Lovelock, J. Math. Phys. 12 (1971) 498.

[2] R. Troncoso and J. Zanelli, Class. Quant. Grav. 17, 4451 (2000) arXiv:hep-th/9907109.

[3] J. Crisostomo, R. Troncoso and J. Zanelli, Phys. Rev. D 62, 084013 (2000) arXiv:hep-th/0003271.

[4] A. H. Chamseddine, Phys. Lett. B 233, 291 (1989).

[5] R. Aros, M. Contreras, Phys.Rev. D73 (2006) 087501.

[6] F. Canfora, A. Giacomini and S. Willison, "Some exact solutions with torsion in 5-D Einstein-Gauss-Bonnet gravity", arXiv:0706.2891 [gr-qc]. To be published in Phys. Rev. D.

[7] G. Dotti, J. Oliva and R. Troncoso, "Exact solutions for the Einstein-GaussBonnet theory in five dimensions: Black holes, wormholes and spacetime horns", arXiv:0706.1830 [hep-th].

[8] R. G. Cai and K. S. Soh, Phys. Rev. D 59, 044013 (1999).

[9] R. Aros, R. Troncoso and J. Zanelli, Phys. Rev. D 63, 084015 (2001).

[10] D. G. Boulware and S. Deser, Phys. Rev. Lett. 55, 2656 (1985). 
[11] M. Banados, C. Teitelboim and J. Zanelli, Phys. Rev. D 49, 975 (1994).

[12] R. G. Cai, Phys. Rev. D 65, 084014 (2002) arXiv:hep-th/0109133]. See also R. G. Cai and Q. Guo, Phys. Rev. D 69, 104025 (2004) [arXiv:hep-th/0311020].

[13] M. Banados, C. Teitelboim and J. Zanelli, Phys. Rev. Lett. 69, 1849 (1992) arXiv:hep-th/9204099.

[14] M. Banados, M. Henneaux, C. Teitelboim and J. Zanelli, Phys. Rev. D 48 (1993) 1506 arXiv:gr-qc/9302012.

[15] O. Coussaert and M. Henneaux, Phys. Rev. Lett. 72, 183 (1994) arXiv:hep-th/9310194.

[16] A. Achucarro and P. K. Townsend, Phys. Lett. B 180, 89 (1986).

[17] R. Aros, C. Martinez, R. Troncoso and J. Zanelli, JHEP 0205, 020 (2002) arXiv:hep-th/0204029.

[18] A. H. Chamseddine, Nucl. Phys. B 346, 213 (1990).

[19] R. Troncoso and J. Zanelli, Int. J. Theor. Phys. 38, 1181 (1999) arXiv:hep-th/9807029.

[20] O. Miskovic, R. Troncoso and J. Zanelli, Phys. Lett. B 637, 317 (2006) arXiv:hep-th/0603183.

[21] J. A. Wolf, Spaces of constant curvature, ch. 2, p. 69. Publish or Perish, Wilmington, Delaware (USA), fifth ed., 1984.

[22] O. Chandia, R. Troncoso and J. Zanelli, "Dynamical content of Chern-Simons supergravity", arXiv:hep-th/9903204.

[23] M. Banados, Phys. Lett. B 501, 150 (2001) arXiv:hep-th/0009066.

[24] R. S. Garavuso and F. Toppan, "Chern-Simons AdS_5 supergravity in a RandallSundrum background", arXiv:0705.4082 [hep-th].

[25] F. Canfora, "Some solutions with torsion in Chern-Simons gravity and observable effects," arXiv:0706.3538 [gr-qc].

[26] M. Banados, O. Miskovic and S. Theisen, JHEP 0606, 025 (2006) arXiv:hep-th/0604148.

[27] A. Giacomini, R. Troncoso and S. Willison, Class. Quant. Grav. 24, 2845 (2007) arXiv:hep-th/0610077. 
[28] A. A. Garcia, F. W. Hehl, C. Heinicke and A. Macias, Phys. Rev. D 67, 124016 (2003) [arXiv:gr-qc/0302097].

[29] R. Troncoso and J. Zanelli, Phys. Rev. D 58, 101703 (1998) arXiv:hep-th/9710180. 